Eric Schmidt. PayPal's co-founder Peter Thiel has bet on Transcriptic, of Menlo Park, California, and Emerald Cloud Lab, of South San Francisco, California, both of which are robotic cloud-based research service providers, as well as Emeryville-based Bolt Threads, a company making materials from spider-silk fibers. At the SynbiTECH conference in London in June, Cumbers noted that the field amassed a record \$1.9 billion in venture funding in 2018, with a similar investment figure expected for 2019.

Station B will follow the framework that synthetic biology normally applies to designing and building biological systems: 'design-build-test-learn'. The design phase uses programming languages Microsoft developed to represent information about a cell's metabolism, genetics, and transcription, regulation and signaling networks. The build phase converts those designs into DNA sequences and experiment instructions The wet-lab experiments that follow will be carried out not by human researchers operation by trial and error, but by Synthace's ANTHA robotic platform. Machine-learning algorithms will then analyze the results and use those experiments to refine subsequent designs and increase reproducibility. By operating with cloud-based algorithms and automated experimentation, scientists can use the platform to improve their efficiency and work more reliably.

As yet, Station B is a research project rather than a commercial offering, but the Microsoft Research group anticipates that the pilot projects with Oxford Biomedica and Princeton's Bassler will prove useful to its customers in both academic and large-scale industrial settings, says Andrew Phillips, head of the Biological Computation Group at Microsoft Research in Cambridge, UK.

For the most part, when synthetic biology labs want to predict, say, the best conditions for a new drug, or a cell's response to an RNA sequence, they rely on computational models of cells and cell components to run simulations. Such models need to be detailed and accurate enough for researchers to successfully design new and modified organisms in silico. And that requires extraordinary amounts of data about the cells, says Phillips. Biotech companies resort to brute force to build them-typically testing many biomolecular variations in parallel. "We can't get away from that completely but we're really pushing hard on learning computational models from the data using advanced machine-learning techniques," he says. Key to building those models is gaining a detailed understanding of cellular components, such as organelles, microtubules and cytoskeletal proteins. "So you would build a component, you'd use it in one context, you get measurements, you characterize it, use it in another context [where] it may behave slightly differently," he says. "You learn over time about which properties are intrinsic to the component and which properties depend on the environment."

Rapid knowledge generation is one of the main selling points for AI-driven synthetic biology platforms such as System B. For a company, gaining such insights can boost productivity in the short term by narrowing the field of candidates, for example, among biomolecular variants, and in the long term by contributing to mechanistic cell models that enable rational cell design.

Though machine learning is key to generating knowledge, the strength of platforms like Station B is that they tie all the processes together, from designing cells to automating wet lab functions to analyzing test results via machine-learning software, says Nathan Hillson, a synthetic biologist and bioinformatician with the US Department of Energy and cofounder and chief scientific officer of San Francisco-based TeselaGen, an AI-driven, cloud-based platform for biological systems. It also matters that researchers use the platforms in a way that produces highquality, reproducible data, he says. "It's the whole engineering cycle, so everything from the original design of experiment to actually figuring out how to make those designs in the real world: how to fabricate them, how to validate that they're made correctly, how do you test them, how do you capture that test data, how do you learn from that test data to then inform your next round of design," he says. "Unless you have software infrastructure coordinated with automation and analytical instrumentation, and all of those types of things across that whole cycle, you can't really iterate effectively and quickly."

Oxford Biomedica's primary goal in working with Microsoft is to reduce the cost of producing a dose of lentiviral vector, says Jason Slingsby, the company's chief business officer. Oxford Biomedica is a cell and gene therapy biotech known in the industry for providing lentiviral vectors to numerous biotech and pharma companies. Oxford Biomedica produces for Novartis the chimeric antigen receptor (CAR)-T therapy Kymriah, which is approved for children and young adults with acute lymphoblastic leukemia and adults with diffuse large B-cell lymphoma. It's an expensive therapy: $\$ 475,000$ for the leukemia therapy and $\$ 373,000$ for the lymphoma therapy in the United States. "By lowering the price we might have a chance to democratize access to gene therapy treatments," says Slingsby. "It also helps global supply."

\section{Pfizer lured by Array's cancer know-how}

Pfizer announced June 17 that it will purchase Array BioPharma for \$11.4 billion. In return for its big-bucks outlay, Pfizer gains the cancer combination Braftovi and Mektovi (encorafenib and binimetinib), marketed for the treatment of melanoma and in use in approximately 30 clinical trials, as well as potential royalty streams from Array's out-licensed clinical programs. Several Array-initiated programs using these drugs in combinations focus on colorectal cancer. For example, Braftovi, Mektovi and Erbitux (cetuximab, Eli Lilly) are in a phase 3 study, and Mektovi plus the PD-1 inhibitor Opdivo (nivolumab, Bristol-Myers Squibb) are in a phase 2 trial. Positive results could mean that Pfizer is able to benefit from its bolt-on biotech purchase to gain an advantage in the not-so-crowded colorectal cancer market. Pfizer will also get its hands on two of Array's drug discovery platforms it deemed "highly productive" during the conference call announcing the acquisition. Array's first platform is a cell-based phenotypic screen for kinase inhibitors, together with a chemogenomic library of kinase inhibitors that includes chemoinformatic tools to deconvolute screening results back to the molecular targets of the inhibitors. The kinase inhibitor platform has been a rich source of clinical-stage kinase inhibitors, leading Array to strike numerous deals. Among the programs originated by Array's platform are the kinase inhibitors Vitrakvi (larotrectinib, licensed to Bayer), selumetinib (AstraZeneca), tucatinib (Seattle Genetics), ipatasertib (Genentech) and varlitinib (Aslan). Array's second platform is a target-based discovery tool that uses knowledge of cocrystal structures to identify the optimal modulation site on traditionally difficult-to-drug targets such as Ras. An example is MRTX849 (Mirati Therapeutics), currently in earlystage trials for KRAS-mutant solid tumors. Pfizer sees these platforms as a potentially fruitful source of new molecules, with the potential for "one investigational new drug per year ... starting possibly 2019," according to Pfizer executive Mikael Dolsten in the conference call. He added that new molecules might be combined with Pfizer drugs such as the PD-1 inhibitor sasanlimab.

Published online: 2 August 2019 https://doi.org/10.1038/s41587-019-0221-0 